\title{
Transport anomalies in the layered compound $\mathrm{BaPt}_{4} \mathrm{Se}_{6}$
}

\author{
Sheng $\mathrm{Li}^{1}$, Yichen Zhang $\mathbb{D D}^{2}$, Hanlin $\mathrm{Wu}^{1}$, Huifei Zhai ${ }^{1}$, Wenhao Liu ${ }^{1}$, Daniel Peirano Petit ${ }^{1}$, Ji Seop Oh ${ }^{2,3}$, Jonathan Denlinger ${ }^{4}{ }^{4}$, \\ Gregory T. McCandless ${ }^{5}$, Julia Y. Chan $\mathbb{D}^{5}$, Robert J. Birgeneau ${ }^{3,6}$, Gang Li $\mathbb{D}^{7}$, Ming Yi $\mathbb{D}^{2 \times}$ and Bing Lv(D)
}

We report a layered ternary selenide $\mathrm{BaPt}_{4} \mathrm{Se}_{6}$ featuring sesqui-selenide $\mathrm{Pt}_{2} \mathrm{Se}_{3}$ layers sandwiched by $\mathrm{Ba}$ atoms. $\mathrm{The}_{2} \mathrm{Pe}_{3}$ layers in this compound can be derived from the Dirac-semimetal $\mathrm{PtSe}_{2}$ phase with Se vacancies that form a honeycomb structure. This

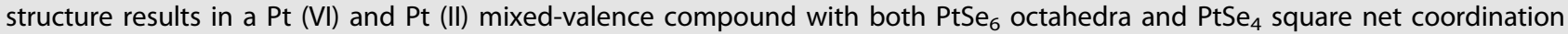
configurations. Temperature-dependent electrical transport measurements suggest two distinct anomalies: a resistivity crossover, mimic to the metal-insulator (M-I) transition at $\sim 150 \mathrm{~K}$, and a resistivity plateau at temperatures below $10 \mathrm{~K}$. The resistivity crossover is not associated with any structural, magnetic, or charge order modulated phase transitions. Magnetoresistivity, Hall, and heat capacity measurements concurrently suggest an existing hidden state below $5 \mathrm{~K}$ in this system. Angle-resolved photoemission spectroscopy measurements reveal a metallic state and no dramatic reconstruction of the electronic structure up to $200 \mathrm{~K}$.

npj Quantum Materials (2021)6:80; https://doi.org/10.1038/s41535-021-00382-x

\section{INTRODUCTION}

Two-dimensional (2D) transition metal dichalcogenides (TMDs), with various polytype structures such as $1 \mathrm{~T}, 1 \mathrm{~T}^{\prime}, 2 \mathrm{H}$, and $3 \mathrm{R}$ phases, have provided a fertile ground for fundamental quantum materials research and emergent potential applications in the past decade due to the fascinating physical properties discovered in these materials ${ }^{1-17}$. In most cases, structural defects such as vacancies and grain boundaries disrupt the translational symmetry of these pristine lattices and significantly impact their physical and chemical properties. Typically, these defects are detrimental to the carrier mobility and associated charge transport performance ${ }^{18-20}$. However, defect engineering through carefully controlled atomic defects has recently emerged as a versatile and effective tool that can significantly improve the physical properties, tune the electronic structures, and tailor their device performances for 2D materials. It has been demonstrated that different types of controlled defect structures have led to the enhancement of electrical transport, optical, and chemical properties, for various TMD materials ${ }^{21-23}$. If such defects are well-ordered, a new type of structure or even unprecedented physical properties could also be developed ${ }^{24,25}$. For example, $\mathrm{Mo}_{6} \mathrm{Te}_{6}$ nanowire forms at the boundaries of $2 \mathrm{H}-\mathrm{MoTe}_{2}$ through thermal annealing under vacuum ${ }^{26}$; novel $\mathrm{Mo}_{2} \mathrm{~S}_{3}$ is fabricated through a periodic assembly of chalcogen vacancy lines in the corresponding $\mathrm{MoS}_{2}$ monolayers ${ }^{27}$. Point defects and line defects of chalcogen atoms induce magnetism in $\mathrm{PtSe}_{2}$ and $\mathrm{ReS}_{2}$ where magnetism does not exist in the pristine materials ${ }^{28,29}$. Besides defect engineering, chemical intercalation that introduces guest species into the van der Waals gaps can also effectively change the band filling and the chemical potential of TMD materials and lead to drastic changes in their electronic, transport, and optical properties ${ }^{30-32}$. These intercalates can range from cations, anions, neutral atoms, and even organic molecules, and could also introduce charge orders ${ }^{33,34}$, superconductivity $^{35,36}$, or magnetic orders ${ }^{37,38}$ into the host TMD materials.
Among the known TMDs, $\mathrm{PtSe}_{2}$ has been well-known for its high performance in photocatalysis, electrocatalysis, and high mobility in field-effect transistors ${ }^{39-45}$. With dimension reduction of $\mathrm{PtSe}_{2}$, the physical properties will change from that of a bulk Dirac-semimetal to a monolayer semiconductor with an indirect bandgap of $1.2 \mathrm{eV}^{46}$. Different types of defects have been found in the bulk and thin films of $\mathrm{PtSe}_{2}$, and they have experimentally and theoretically impacted the related properties ${ }^{28,47}$. Thicknessindependent semiconducting-to-metallic conversion, in contrast to semiconducting-to-metal transition with reduced thickness, has been observed through plasma-driven atomic defect engineering in $\mathrm{PtSe}_{2}$ films ${ }^{48}$. Theoretical calculations suggested that large spinorbit splitting can be induced by introducing point defects in the $\mathrm{PtSe}_{2}{ }^{49}$, and the Se vacancy line defects in monolayer $\mathrm{PtSe}_{2}$ could cause a sizable spin splitting in the defect states ${ }^{50}$. This large spinorbital splitting gives rise to persistent spin textures, which protect the spin from decoherence and induce an extraordinarily long spin lifetime for designing spintronic devices.

As a part of chemical doping studies with controlled defects synthesis for bulk $\mathrm{PtSe}_{2}$ materials ${ }^{51}$, we report herein a ternary compound of $\mathrm{BaPt}_{4} \mathrm{Se}_{6}$ of layered structure featured with sesquiselenide $\mathrm{Pt}_{2} \mathrm{Se}_{3}$ layers sandwiched by $\mathrm{Ba}$ atoms. Each sesquiselenide $\mathrm{Pt}_{2} \mathrm{Se}_{3}$ layer could be considered as a $\mathrm{PtSe}_{2}$ structure with ordered Se vacancies. It has an atomic coordination within the structure of both the $\mathrm{PtSe}_{6}$ octahedra and the $\mathrm{PtSe}_{4}$ square planar, which results in a mixed-valence compound with Pt (II):Pt (IV) = $3: 1$. It is also interesting to note that the Se atom framework in this compound could be viewed as stacks of distorted Kagome nets formed by Se atoms. We observe a resistivity crossover, mimic to the metal-insulator transition $\sim 150 \mathrm{~K}$ and a resistivity plateau at temperatures below $10 \mathrm{~K}$. The $\mathrm{X}$-ray single-crystal diffraction, temperature-dependent electrical resistivity, magnetoresistivity, Hall, effect, heat capacity, and angle-resolved photoemission spectroscopy (ARPES) measurements are carried out to fully characterize this compound and understand the intriguing transport anomalies observed in this system.

\footnotetext{
${ }^{1}$ Department of Physics, The University of Texas at Dallas, Richardson, TX 75080, USA. ${ }^{2}$ Department of Physics and Astronomy, Rice University, Houston, TX 77005, USA. ${ }^{3}$ Department of Physics, University of California, Berkeley, CA 94720, USA. ${ }^{4}$ Advanced Light Source, Lawrence Berkeley National Laboratory, Berkeley, CA 94720, USA. ${ }^{5}$ Department of Chemistry, The University of Texas at Dallas, Richardson, TX 75080, USA. ${ }^{6}$ Materials Science Division, Lawrence Berkeley National Laboratory, Berkeley, CA 94720, USA. ${ }^{7}$ School of Physical Science and Technology, Shanghai Tech University, Shanghai 200031, China. ${ }^{\varpi_{e}}$ mail: mingyi@rice.edu; blv@utdallas.edu
} 
Table 1. Crystal structure of $\mathrm{BaPt}_{4} \mathrm{Se}_{6}$ at room temperature and $100 \mathrm{~K}$.

\begin{tabular}{|c|c|c|c|c|c|c|}
\hline Temperature & \multicolumn{3}{|l|}{$300 \mathrm{~K}$} & \multicolumn{3}{|l|}{$100 \mathrm{~K}$} \\
\hline Space group & \multicolumn{3}{|l|}{ C2/c (no. 15) } & \multicolumn{3}{|l|}{ C2/c (no. 15) } \\
\hline$b$ & \multicolumn{3}{|l|}{$7.406(2) \AA$} & \multicolumn{3}{|l|}{ 7.4004(14) $\AA$} \\
\hline c & \multicolumn{3}{|l|}{$12.461(3) \AA$} & \multicolumn{3}{|l|}{$12.406(2) \AA$} \\
\hline$\beta$ & \multicolumn{3}{|l|}{$118.089(7)^{\circ}$} & \multicolumn{3}{|l|}{$118.374(8)^{\circ}$} \\
\hline \multicolumn{7}{|l|}{ Atomic position } \\
\hline $\operatorname{Ba} 1(4 c)$ & $1 / 4$ & $3 / 4$ & $1 / 2$ & $1 / 4$ & $3 / 4$ & $1 / 2$ \\
\hline $\operatorname{Pt} 1(4 e)$ & 0 & $0.35664(5)$ & $1 / 4$ & 0 & $0.35783(4)$ & $1 / 4$ \\
\hline $\operatorname{Pt} 2(4 e)$ & 0 & $0.85699(5)$ & $1 / 4$ & 0 & $0.85812(4)$ & $1 / 4$ \\
\hline $\mathrm{Pt} 3(8 f)$ & $0.25059(2)$ & $0.59819(3)$ & $0.24539(3)$ & $0.25057(2)$ & $0.59922(3)$ & $0.24530(2)$ \\
\hline$\theta$ range & \multicolumn{3}{|l|}{$3.30-30.57^{\circ}$} & \multicolumn{3}{|l|}{$3.30-30.54^{\circ}$} \\
\hline Independent reflections & \multicolumn{3}{|l|}{1599} & \multicolumn{3}{|l|}{1575} \\
\hline Refine Parameters & \multicolumn{3}{|l|}{54} & \multicolumn{3}{|l|}{54} \\
\hline $\mathrm{R}$ (int) & \multicolumn{3}{|l|}{0.0564} & \multicolumn{3}{|l|}{0.0458} \\
\hline Final $\mathrm{R}$ indices & \multicolumn{3}{|c|}{$\mathrm{R}_{1}=0.027, \mathrm{wR}_{2}=0.058$} & \multicolumn{3}{|c|}{$\mathrm{R}_{1}=0.022, \mathrm{wR}_{2}=0.055$} \\
\hline Goodness-of-fit & \multicolumn{3}{|l|}{1.056} & \multicolumn{3}{|l|}{1.076} \\
\hline
\end{tabular}

\section{RESULTS AND DISCUSSIONS}

\section{$X$-ray diffraction and structure description}

The details of the $X$-ray single-crystal refinements and the associated crystallographic parameters for $\mathrm{BaPt}_{4} \mathrm{Se}_{6}$ at both $300 \mathrm{~K}$ and $100 \mathrm{~K}$ are provided in Table 1. Additional atomic coordination, selected interatomic distances, angles, and precession images are presented in the supplementary information. In sharp contrast to $\mathrm{PtSe}_{2}$ in the space group of $P \overline{3} m 1, \mathrm{BaPt}_{4} \mathrm{Se}_{6}$ crystallizes in a distinct structure type ( $m C 44)$ and the centrosymmetric monoclinic space group $C 2 / c$ (\#15) with lattice parameters $a=12.715$ (4) $\AA, b=7.406$ (2) $\AA, c=12.461$ (3) $\AA$, and $\beta=118.089$ $(7)^{\circ}$. The X-ray diffraction pattern with peaks in the preferred orientation is shown in Fig. 1a, together with a photograph of the crystals on the $\mathrm{mm}$ scale grid. The crystal structure (Fig. 1b) consists of three distinct Pt sites (Pt1 and Pt2 atoms are at 4e sites and $\mathrm{Pt} 3$ atoms are at the general position $8 f$, as labeled in Fig. $1 \mathrm{~b}$ ). Pt1 atoms at the $4 e$ site are octahedrally coordinated with six neighboring Se atoms and have a formal $\mathrm{Pt}^{4+}$ valence. The Pt2 atoms at $4 e$ site and the Pt3 atoms at the $8 f$ site, have a square planar environment with nearby Se atoms, and have a formal $\mathrm{Pt}^{2+}$ valence (Supplementary Fig. 1). The $\mathrm{PtSe}_{6}$ octahedra formed by Pt1 atoms are edge shared with the square planar $\mathrm{PtSe}_{4}$ formed by both Pt2 and Pt3 atoms (Fig. 1b). Each PtSe ${ }_{6}$ octahedra is surrounded by six $\mathrm{PtSe}_{4}$ square planar, and each $\mathrm{PtSe}_{4}$ square planar is shared by two $\mathrm{PtSe}_{6}$ octahedra, together they form a sesqui-selenide $\mathrm{Pt}_{2} \mathrm{Se}_{3}$ layer structure with the stacking along the $c$ axis. The interlayer Se-Se distance is $3.478 \AA$, suggesting weak van der Waals interactions between the sesqui-selenide layers. The Ba atoms are sandwiched between layers and can be chargebalanced as $\left(\mathrm{Ba}^{2+}\right)\left(\mathrm{Pt}^{2+}\right)_{3}\left(\mathrm{Pt}^{4+}\right)\left(\mathrm{Se}^{2-}\right)_{6}$ with $\mathrm{Pt}(\mathrm{II}): \mathrm{Pt}(\mathrm{IV})=3: 1$. The Pt (II)-Se distances in the square planar configuration range from 2.4371 (9) $\AA$ to 2.4704 (9) $\AA$, and are generally shorter than the Pt(IV)-Se distances with octahedral configurations [2.5008(9) $\AA$ to 2.5169(9) $\AA$ ]. Both the $\mathrm{PtSe}_{6}$ octahedra and $\mathrm{PtSe}_{4}$ square planar are highly distorted resulting from the monoclinic symmetry of the crystal structures (Supplementary Fig. 1 and Supplementary Tables 1 and 2).

The sesqui-selenide $\mathrm{Pt}_{2} \mathrm{Se}_{3}$ layer in this $\mathrm{BaPt}_{4} \mathrm{Se}_{6}$ compound could be considered as the Se-defect-ordered structure of TMD $\mathrm{PtSe}_{2}$ layers. Figure $1 \mathrm{c}$ represents the projection of the $\mathrm{Pt}_{2} \mathrm{Se}_{3}$ slab in $\mathrm{BaPt}_{4} \mathrm{Se}_{6}$. In comparison with the projected monolayer $\mathrm{PtSe}_{2}$ slab (Fig. 1d), one-third of the Se atomic positions in the $\mathrm{Pt}_{2} \mathrm{Se}_{3}$ slab remain vacant, as highlighted by red open circles, and the vacancies are alternatively shifted to form a well-ordered honeycomb defect pattern. This ordered vacancy pattern is apparently different from the linear vacancy line pattern observed in the $\mathrm{Mo}_{2} \mathrm{~S}_{3}$ structures and suggests a likely significant change in the electronic structures and transport properties compared with the parent compound $\mathrm{PtSe}_{2}$.

\section{Electrical transport data and discussions}

The temperature-dependent resistivity data of the $\mathrm{BaPt}_{4} \mathrm{Se}_{6}$ are shown in Fig. 2a. In sharp contrast to the overall semi-metallic behavior of the bulk $\mathrm{PtSe}{ }_{2}$, this vacancy-ordered sesqui-selenide shows a drastic resistivity crossover at $\sim 150 \mathrm{~K}$ with metallic behavior from room temperature down to $150 \mathrm{~K}$ and semiconducting behavior below $150 \mathrm{~K}$. Using the thermal activation model formula of $\rho \propto e^{E_{a} / k_{B} T}$ within the temperature range from $150 \mathrm{~K}$ to $90 \mathrm{~K}$, we obtain an activation energy of $0.82 \mathrm{meV}$. However, the resistivity begins to deviate from the thermal activation model below $80 \mathrm{~K}$, starts to saturate below $20 \mathrm{~K}$, and reaches a plateau between $10 \mathrm{~K}$ and $2 \mathrm{~K}$, reminiscent of the resistivity plateau observed in $\mathrm{SmB}_{6}$.

Both the resistivity crossover at $150 \mathrm{~K}$ and resistivity plateau at lower temperatures are quite intriguing, as they hint at a number of potential causes ranging from a magnetic phase transition, charge density wave (CDW) transition, Kondo effect, topological effects, and localization. We, therefore, carried out temperaturedependent magnetization, single-crystal $\mathrm{X}$-ray diffraction, and heat capacity measurements to further examine the origins of 


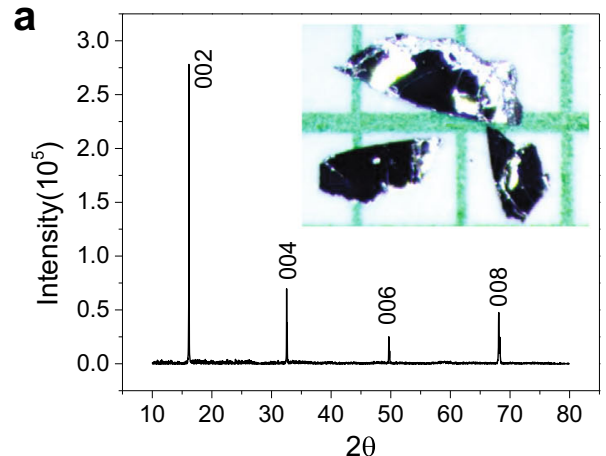

b
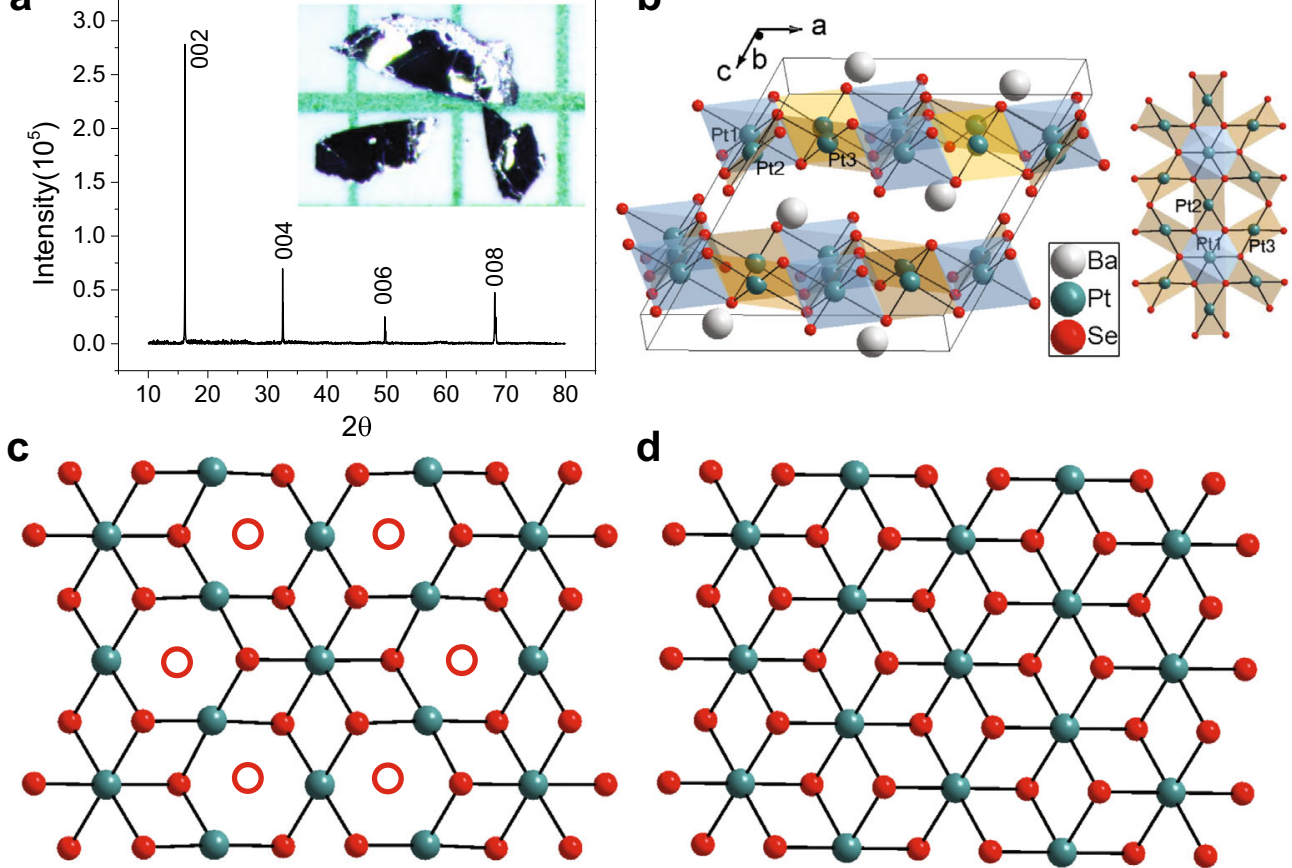

Fig. 1 Structural analysis of the $\mathrm{BaPt}_{4} \mathrm{Se}_{6}$. a Powder X-ray diffraction on single-crystal with the preferred orientation along $c$ axis, inset is the optical image of the $\mathrm{BaPt}_{4} \mathrm{Se}_{6}$ single crystals. b Side view of $\mathrm{BaPt}_{4} \mathrm{Se}_{6}$ structure, with the $\mathrm{PtSe}_{6}$ octahedra and the adjacent $\mathrm{PtSe} \mathrm{e}_{4}$ square planar. c Projection of the $\mathrm{Pt}_{2} \mathrm{Se}_{3}$ layer, with the red open circles, denote the vacancy of Se atoms and compare to $\mathbf{d}$ top view of the PtSe $\mathrm{e}_{2}$ layer from $1 T-\mathrm{PtSe}_{2}$.
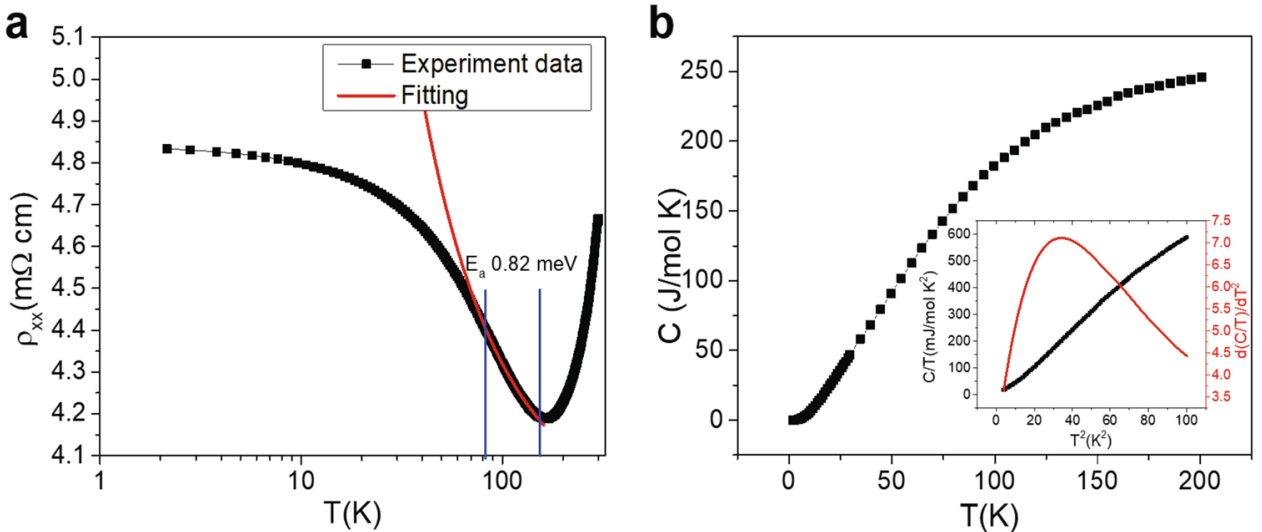

Fig. 2 Electrical resistivity and heat capacity data. a Temperature-dependent resistivity in log scale to show the low-temperature resistivity saturation, the red solid line is the fitted data using the activation energy model. b Heat capacity of $\mathrm{BaPt}_{4} \mathrm{Se}_{6}$ from $2 \mathrm{~K}$ to $200 \mathrm{~K}$ where no clear anomalies or jumps are observed, and the inset is the low-temperature heat capacity and first derivative (in red) data from $2 \mathrm{~K}$ to $10 \mathrm{~K}$.

these transport anomalies. First, no magnetic transition is observed from temperature-dependent magnetization measurement (Supplementary Fig. 4). This is consistent with the electron configuration of $5 d^{8}$ for $\mathrm{Pt}$ (II) atoms in the square planar coordination and $5 d^{6}$ for $\mathrm{Pt}$ (IV) atoms in the octahedral coordination as for both configurations the $5 d$ electrons are fully paired. In addition, there are no magnetic ions in this system. Hence, resistivity anomalies cannot be induced by the Kondo effect. Secondly, no clear structural transformation is observed from X-ray single-crystal diffraction in the low-temperature regime down to $100 \mathrm{~K}$. The overnight low-temperature $\mathrm{X}$-ray single-crystal diffraction at $100 \mathrm{~K}$ is collected and the refined crystal structures are shown in Table 1. Clearly, no symmetry changes nor splitting of Wyckoff positions is observed between $100 \mathrm{~K}$ and $300 \mathrm{~K}$. The integrated precession images that represent the reciprocal space diffraction spots (Supplementary Fig. 2) also do not reflect any
CDW-modulated structural distortions at $100 \mathrm{~K}$ compared with the refined structural model at $300 \mathrm{~K}$ shown in Table 1. In addition, no clear jumps or anomalies are observed from heat capacity data between $2 \mathrm{~K}$ and $200 \mathrm{~K}$ (Fig. 2b), which further supports that no structure transition nor other first-order phase transitions exist in this system. Therefore, electron localization is the most likely cause of the resistivity upturn at $150 \mathrm{~K}$ in this system. A combination of the thermal activation model and variable range hopping model using different dimension indices to represent the strong localization effect has been used to fit the data between $80 \mathrm{~K}$ and $20 \mathrm{~K}$, and yield unreasonably small activation energy $<1 \mu \mathrm{eV}$. This suggests that weak localization rather than strong localization exists in the system.

Regarding the origin of the resistivity plateau observed at low temperatures, magnetic effects such as the Kondo effect or magnetic field-induced resistivity saturation as observed in $\mathrm{WTe}_{2}$ 

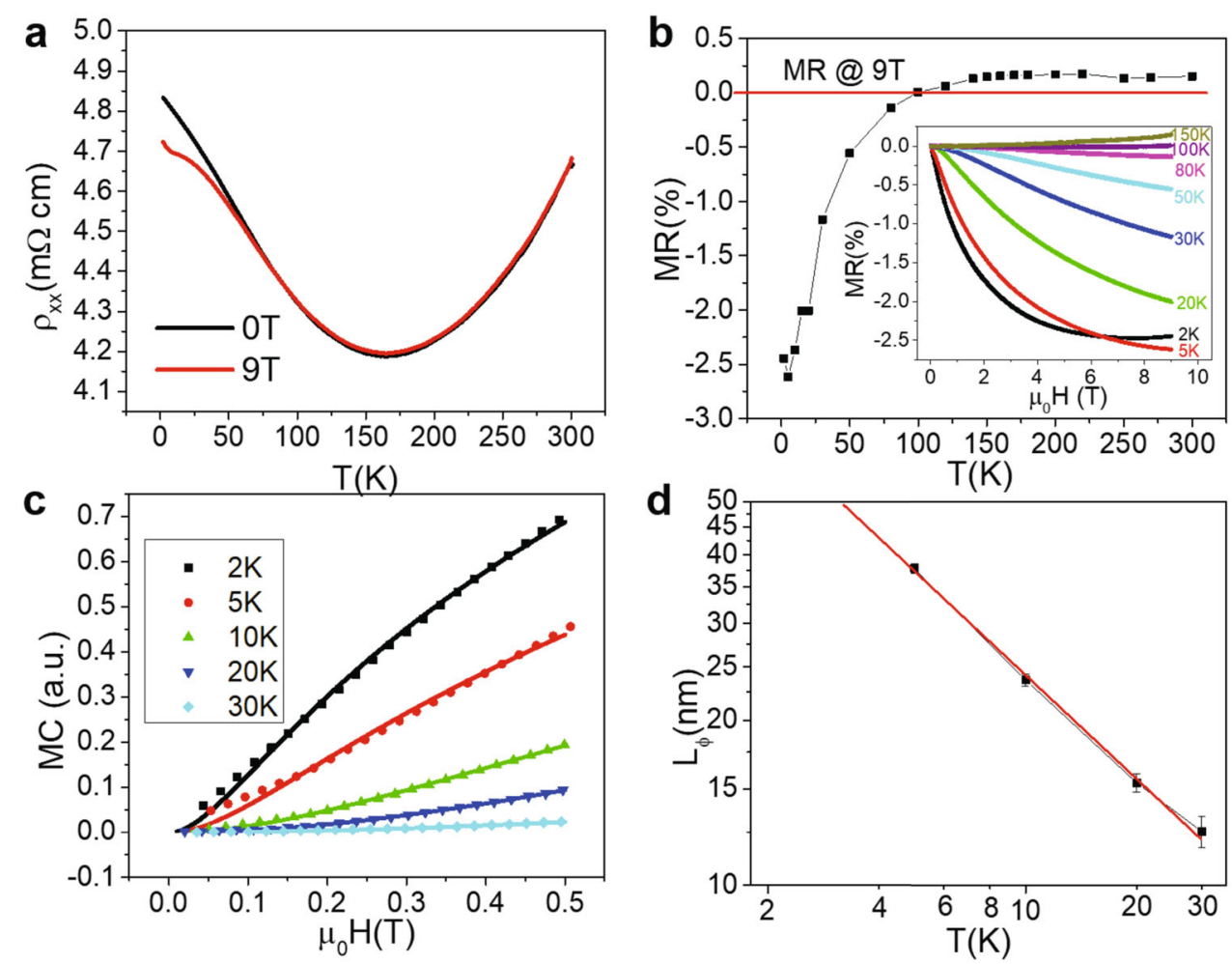

Fig. 3 In-plane magnetoresistivity data and related analysis. a Temperature-dependent in-plane resistivity data at zero magnetic field and with the applied magnetic field up to $9 \mathrm{~T}$. b Magnetoresistivity at different temperatures under $9 \mathrm{~T}$. The inset shows the isothermal magnetic field-dependent resistivity behavior. c Magnetoconductance at low magnetic field range fitted by the HLN formula (solid line) at several temperatures, and $\mathbf{d}$ Temperature-dependent phase coherence length at different temperatures derived from the HLN formula with linear fitting in the double log scales.

system are not applicable here. One possible explanation could be the contribution of some additional metallic states, which could originate from multiple conducting channels or surface states competing with the localization effects at low temperatures.

The low-temperature range heat capacity data are shown in the inset of Fig. 2b. Interestingly, the heat capacity does not exhibit a linear relationship of $C / T$ vs $T^{2}$ following the Debye model $C=\gamma_{N} T$ $+\beta T^{3}$ between $2 \mathrm{~K}$ and $10 \mathrm{~K}$. By taking the first-order derivative of the nonlinear behavior from $2 \mathrm{~K}$ to $10 \mathrm{~K}$, we can clearly observe a broad peak with a maximum at $\sim 6 \mathrm{~K}$. This peak may suggest an existing hidden order in the $\mathrm{BaPt}_{4} \mathrm{Se}_{6}$ system, which could be responsible for the resistivity plateau observed from the electrical resistivity measurement.

Resistivities with and without applying a magnetic field at $9 \mathrm{~T}$ in the whole temperature range are shown in Fig. 3a. A clear change of magnetoresistance sign is observed at $\sim 100 \mathrm{~K}$, from negative in the low-temperature range to positive in the high-temperature range. The isothermal magnetoresistivity up to $9 \mathrm{~T}$ at different temperatures is shown in Fig. 3b, which is consistent with Fig. 3a. Three small yet non-negligible anomalies could be observed: (i) a broad hump at $150 \mathrm{~K}$, which is on par with the M-I transition temperature observed in Fig. 2a; (ii) a change of sign of the magnetoresistance at $100 \mathrm{~K}$ from positive to negative is observed; (iii) the magnetoresistance upturn below $5 \mathrm{~K}$. As the localization is typically induced by disorder, the broad hump at $150 \mathrm{~K}$ could be explained by the enhanced scattering caused by disorder under magnetic field. However, at low temperatures, a negative magnetoresistivity owing to the localization effect will emerge and become stronger with decreasing temperature. The positive and negative magnetoresistance will compensate each other at intermediate temperatures, which could explain the significant changes of magnetoresistance at $\sim 100 \mathrm{~K}$. Below $100 \mathrm{~K}$, the negative magnetoresistance induced by localization becomes predominant. Interestingly, an upturn of the magnetoresistivity below $5 \mathrm{~K}$ is observed, which could also be clearly noticed from the inset of Fig. 3b. In comparison with the $5 \mathrm{~K}$ data, the magnetoresistivity at $2 \mathrm{~K}$ bends down at high magnetic fields.

In order to demonstrate the weak localization effect in this system, we convert the magnetoresistance data to the magnetoconductance at a low field range with temperatures far below $100 \mathrm{~K}$ to minimize the influence of the classical magnetoresistance and fit the data using the Hikami-Larkin-Nagaoka (HLN) formula ${ }^{52}$

$\frac{\Delta \sigma(B)}{G_{0}}=a\left[\Psi\left(\frac{1}{2}+\frac{B_{\phi}}{B}\right)-\ln \left(\frac{B_{\phi}}{B}\right)\right]$

which describes the quantum correction to conductivity owing to weak localization. In the equation $G_{0}=e^{2} /\left(2 \pi^{2} \hbar\right)$, $a$ is the parameter in the renormalization group equation, $\psi(x)$ is the digamma function, $B_{\phi}=\hbar /\left(4 e L_{\phi}^{2}\right)$ is the characteristic value of the magnetic field with $L_{\phi}$ as the phase-coherent length. The fitting results are shown in Fig. 3c, where the data generally are described reasonably well by the HLN formula, some deviation of the fits from experimental values are observed at our lowest measured temperature of $2 \mathrm{~K}$ and $5 \mathrm{~K}$, which likely is due to the additional metallic states as seen in the previous resistivity data in Fig. 2a. The phase coherence length $L_{\phi}$ extracted from $B_{\phi}$, changes from $\sim 47 \mathrm{~nm}$ at $2 \mathrm{~K}$ to $\sim 12 \mathrm{~nm}$ at $30 \mathrm{~K}$ (Fig. 3d). As we increase temperature, the phase coherence length decreases, typically following the power-law relationship $L_{\phi} \propto T^{-n}$. As the coherence length value at $2 \mathrm{~K}$ is affected by additional metallic states (Fig. 3c), we therefore only perform a linear fitting from $5 \mathrm{~K}$ to $30 \mathrm{~K}$ (red solid line) (Fig. 3d) and obtain a fitted index of $n=0.62$-a value close to $1 / 2$ indicating the $2 D$ nature of this system.

In order to further understand the carrier contributions at low temperatures for $\mathrm{BaPt}_{4} \mathrm{Se}_{6}$, we performed a Hall measurement (Fig. 4a). By sweeping the magnetic field at different temperatures, 

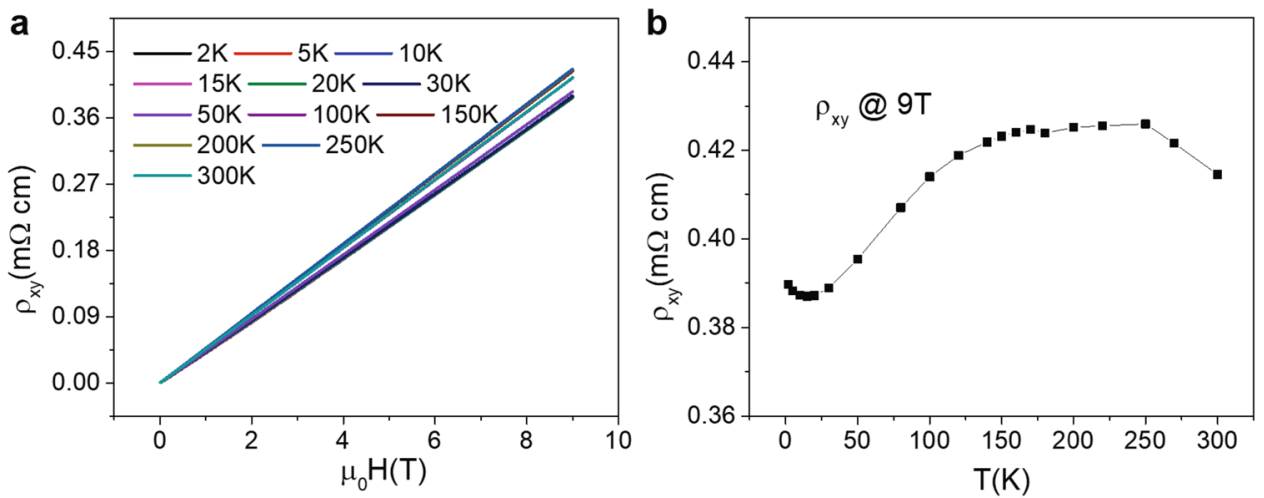

Fig. 4 Hall resistivity data. a Field-dependent Hall resistivity of $\mathrm{BaPt}_{4} \mathrm{Se}_{6}$ at different temperatures up to $9 \mathrm{~T}$. b Temperature-dependent Hall resistivity at $9 \mathrm{~T}$.

a
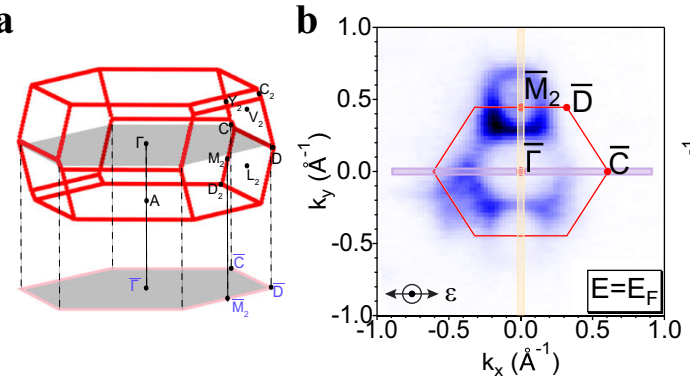

c

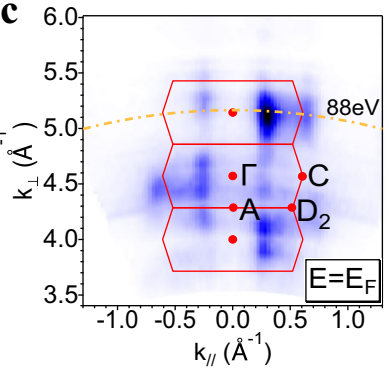

d

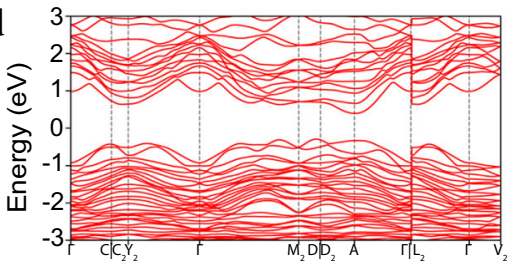

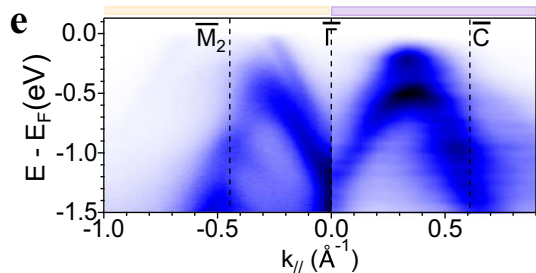

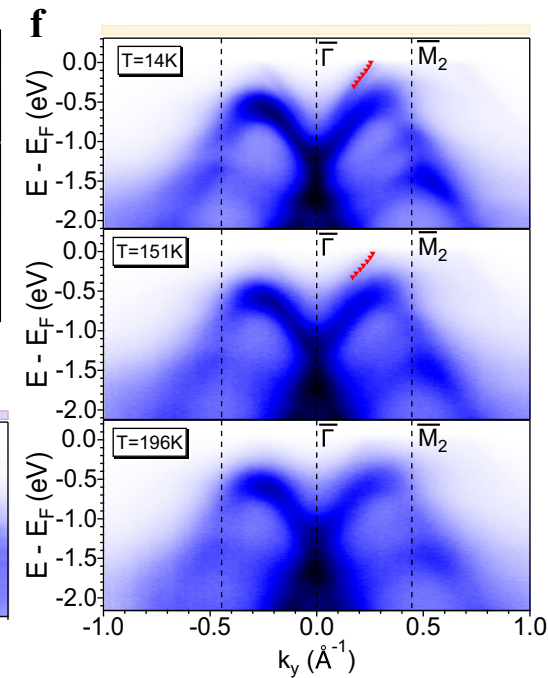

Fig. 5 Angle-resolved photoemission spectroscopy results. a 3D BZ of $\mathrm{BaPt}_{4} \mathrm{Se}_{6}$ where the gray-shaded area denotes the projected (001) surface. b FS map integrated within a $2 \mathrm{meV}$ window of the projected (001) surface using $88 \mathrm{eV} \mathrm{LH}$ photons at $T=13.5 \mathrm{~K}$. Polarization of incident photons is indicated by $\varepsilon$. c FS map along the $k_{z}$ direction. d DFT calculation for bulk $\mathrm{BaPt}_{4} \mathrm{Se}_{6} \mathrm{With}^{\mathrm{SOC}}$ included showing a semiconducting ground state. e Band dispersions along the $\bar{\Gamma}-\bar{M}_{2}$ and $\bar{\Gamma}-\bar{C}$ directions, where the momentum directions have been marked in b. f Band dispersions along the $\bar{\Gamma}-\bar{M}_{2}$ direction at $14 \mathrm{~K}, 151 \mathrm{~K}$, and $196 \mathrm{~K}$, respectively, indicated by a yellow vertical line in panel b. Red triangles extracted from MDCs serve as visual guidance for the metallic states crossing the Fermi level.

the Hall resistivity shows a linear relationship with the magnetic field. The Hall resistivity is positive throughout the whole temperature range, suggesting the predominant hole charge carriers in the system. The absolute Hall resistivity value does not change much in the whole temperature range, and the obtained room temperature hole concentration is on the order of $10^{19}$ $\mathrm{cm}^{-3}$. This is consistent with the resistivity data where no significant change of resistivity is observed despite the $\mathrm{M}-\mathrm{I}$ transition at $150 \mathrm{~K}$. The temperature-dependent Hall resistivity data at $9 \mathrm{~T}$ is shown in Fig. $4 \mathrm{~b}$. Both a broad maximum at $150 \mathrm{~K}$ and a small upturn below $20 \mathrm{~K}$ are observed, consistent with both temperature-dependent resistivity and magnetoresistivity results discussed previously.

\section{Band structure calculations and ARPES measurements}

To visualize the electronic structure of $\mathrm{BaPt}_{4} \mathrm{Se}_{6}$ and its evolution with temperature, we performed ARPES experiments on the highquality single crystals. From density functional theory (DFT) calculations on the nominal crystal structure, $\mathrm{BaPt}_{4} \mathrm{Se}_{6}$ is expected to be a semiconductor (Fig. 5d). The measured dispersions, however, indicate a metallic state. To introduce the measured dispersions, we first show the three-dimensional (3D) Brillouin zone (BZ) notation of $\mathrm{BaPt}_{4} \mathrm{Se}_{6}$ (Fig. $5 \mathrm{a}$ ) and the corresponding projected (001) plane sketched in gray and appended to the measured Fermi surface (FS) in Fig. 5b. Note that due to the low symmetry of the $\mathrm{BaPt}_{4} \mathrm{Se}_{6}$ crystal structure, the FS intensity shows a $C_{2}$ symmetric pattern with respect to the $\bar{\Gamma}-\bar{M}_{2}$ direction only. In the $k_{z}$ mapping displayed in Fig. $5 c$, the FS shows an antisymmetric intensity distribution across $k_{\mathrm{z}}$ of which the apparent periodicity is twice that of the BZ size. Such observed intensity pattern is owing to the structural factor originating from the two stacked $\mathrm{Pt}_{4} \mathrm{Se}_{6}$ layers within each unit cell. Consequently, the $k_{z} \mathrm{FS}$ mapping intensity manifests more strongly the periodicity of the single-layer $\mathrm{Pt}_{4} \mathrm{Se}_{6}$. The measured FS shows Fermi pockets around the $B Z$ boundaries. Along $\bar{\Gamma}-\bar{M}_{2}$, we observe these pockets to be small hole pockets from two linear bands crossing the Fermi level consistent with a metallic nature, albeit near the valence band top (Fig. 5e). From a comparison with the calculation, we observe that the bands away from Fermi level present better consistencies with the calculation. The origin of the mismatch here is likely owing to Se vacancies in the material that slightly hole-dopes the sample. The electronic dispersions of $\mathrm{BaPt}_{4} \mathrm{Se}_{6}$ indicate a degree of 
resemblance to that of $\mathrm{PtSe}_{2}$, albeit with distinction. The dispersions at the $\bar{\Gamma}$ point emerge from an X-shape feature at $~$ $-1.3 \mathrm{eV}$ (Fig. $5 f$ top figure and Supplementary Fig. 5). A pair of bands are observed at the $\bar{M}_{2}$ point, which in $\mathrm{PtSe}_{2}$ are observed to carry spin texture owing to Rashba splitting ${ }^{45}$. However, the measured FS and dispersions show much lower symmetry compared with $\mathrm{PtSe}_{2}$.

We also carried out temperature-dependent measurements to examine the connection to the transport anomalies. The middle panel in Fig. $5 f$ shows the $\bar{\Gamma}-\bar{M}_{2}$ cut at $151 \mathrm{~K}$ corresponding to the $\mathrm{M}-\mathrm{I}$ transition shown above, while the figure at the bottom is taken at a temperature high above the transition. Evidently, considering thermal broadening, major features of the band structure, especially the bands crossing Fermi level remain largely unchanged between $14 \mathrm{~K}$ and $196 \mathrm{~K}$. The red triangles in Fig. $5 \mathrm{f}$ are fitted from momentum distribution curves (MDCs) to highlight the band crossing the Fermi level. Our observation supports the argument that the resistivity crossover $\sim 150 \mathrm{~K}$ is not caused by any change of the density of states near $E_{\mathrm{F}}$, but according to the analysis above can be attributed to the weak localization effect.

In summary, A layered ternary compound $\mathrm{BaPt}_{4} \mathrm{Se}_{6}$ has been discovered with sesqui-selenide layered feature and mix-valence Pt oxidation states. The sesqui-selenide layer is similar to the TMD $\mathrm{PtSe}_{2}$ structure with ordered Se vacancies. Electrical resistivity measurements reveal two transport anomalies: one resistivity crossover at $\sim 150 \mathrm{~K}$ and a resistivity plateau below $10 \mathrm{~K}$. Extensive studies from X-ray diffraction and heat capacities suggest the resistivity crossover is due to weak localization that is related to the structural distortion and ordered honeycomb Se vacancy of the structure. Temperature-dependent ARPES measurements reveal a metallic state between $14 \mathrm{~K}$ and $196 \mathrm{~K}$, and do not show any abrupt electronic changes in the whole temperature range, further supporting the weak localization effect in this compound. Magnetoresistivity, Hall, and heat capacity measurements concurrently suggest an existing hidden state below $5 \mathrm{~K}$, which should be responsible for the resistivity plateau at low temperatures. The hole-like band in the MDC curves from the ARPES measurements is consistent with hole charge carriers from our Hall data. Unfortunately, no clear evidence of the existence of the in-gap state is observed from ARPES measurements down to $14 \mathrm{~K}$, which is slightly higher than the resistivity plateau below $10 \mathrm{~K}$. The exact origin of the emerged metallic ground state at low temperatures is responsible for the resistivity plateau is subject for future studies.

\section{METHODS}

\section{Material synthesis and X-ray diffraction}

The compound was initially discovered during the chemical doping studies for $\mathrm{Ba}_{x} \mathrm{PtSe}_{2}$. The small grain crystals are isolated first for chemical analysis and initial X-ray diffraction studies. The large size of single crystals of $\mathrm{BaPt}_{4} \mathrm{Se}_{6}$ was later directly synthesized through solid-state reaction using Ba pieces (99.9\%, Alfa Aesar), Pt pieces (99\%), and Se shots (99.999\%, Alfa Aesar). A stoichiometric amount of the starting materials were placed into a graphite crucible and then sealed in the fused quartz tube under a vacuum. The tube was placed in a furnace, slowly heat with a rate of $30^{\circ} \mathrm{C} /$ h up to $1100^{\circ} \mathrm{C}$, and maintained for 48 hours before slowly cooled down to $800^{\circ} \mathrm{C}$ with the rate of $3^{\circ} \mathrm{C} / \mathrm{h}$. Thin shinning black crystals can be obtained by carefully cleaving the melt ingot and with preferred $c$ axis orientations (Fig. 1a). The exact crystal structure was determined by Bruker D8 Quest Kappa single-crystal X-ray diffractometer equipped with a Mo Ka I $\mathrm{S}$ microfocus source $(\lambda=0.71073 \AA$ $)$ operating at $50 \mathrm{kV}$ and $1 \mathrm{~mA}$ with a HELIOS optic monochromator and a CMOS detector. The collected data set was integrated with Bruker SAINT and scaled with Bruker SADABS (multiscan absorption correction). The refined chemical composition from single-crystal diffraction was subsequently confirmed by scanning electron microscope energy-dispersive X-ray spectroscopy (Supplementary Fig. 3).

\section{Physical properties measurements}

The electric resistivity and Hall coefficient were measured by employing the standard six probe method using golden wires and silver paste on a $2 \times 1 \times 0.1 \mathrm{~mm}^{3}$ crystal with the temperature down to $2 \mathrm{~K}$ and magnetic field up to $9 \mathrm{~T}$. The magnetoresistivity measurement using standard six probe method with magnetic field perpendicular to the crystal plane and current, the heat capacity measurement using the relaxation method down to $2 \mathrm{~K}$, were performed in a Quantum Design Physical Property Measurement System. ARPES measurements were performed at the MERLIN beamline 4.0.3 at the Advanced Light Source, equipped with a SCIENTA R8000 electron analyzer. The single crystal was cleaved in situ at $13 \mathrm{~K}$ and measured in an ultra-high vacuum with a base pressure better than $5 \times 10^{-11}$ Torr. Photon energy-dependent measurements were carried out from 30 to $120 \mathrm{eV}$, and $88 \mathrm{eV}$ linear horizontal light was selected to obtain the highest data quality.

\section{Electronic structure calculations}

The electronic structure of $\mathrm{BaPt}_{4} \mathrm{Se}_{6}$ was calculated in the framework of DFT within the generalized gradient approximation ${ }^{53}$. The projectoraugmented-wave method implemented in Vienna Ab Initio Simulation Package is employed with the default energy cutoff specified in the pseudopotential file. The momentum grid is taken as $9 \times 9 \times 9$. The SOC is included in the calculation self-consistently. The calculations are performed at the HPC Platform of ShanghaiTech University Library and Information Services and the School of Physical Science and Technology.

\section{DATA AVAILABILITY}

The authors declare that all essential data supporting the findings of this study are available within the paper and its supplementary information.

Received: 23 April 2021; Accepted: 1 September 2021; Published online: 23 September 2021

\section{REFERENCES}

1. Splendiani, A. et al. Emerging photoluminescence in monolayer $\mathrm{MoS}_{2}$. Nano Lett. 10, 1271-1275 (2010).

2. Jaramillo, T. F. et al. Identification of active edge sites for electrochemical $\mathrm{H}_{2}$ evolution from $\mathrm{MoS}_{2}$ nanocatalysts. Science 317, 100-102 (2007).

3. Deng, K. et al. Experimental observation of topological Fermi arcs in type-II Weyl semimetal MoTe 2 . Nat. Phys. 12, 1105-1110 (2016).

4. Wang, Z. et al. MoTe 2 : a type-Il Weyl topological metal. Phys. Rev. Lett. 117, 056805 (2016)

5. Tang, S. et al. Quantum spin Hall state in monolayer $1 T^{\prime}-W T e_{2}$. Nat. Phys. 13, 683-687 (2017).

6. Wang, Y. et al. Structural phase transition in monolayer $\mathrm{MoTe}_{2}$ driven by electrostatic doping. Nature 550, 487-491 (2017).

7. Zhao, C. et al. Enhanced valley splitting in monolayer $\mathrm{WSe}_{2}$ due to magnetic exchange field. Nat. Nanotechnol. 12, 757-762 (2017).

8. Fatemi, V. et al. Electrically tunable low-density superconductivity in a monolayer topological insulator. Science 362, 926-929 (2018).

9. Xu, S.-Y. et al. Electrically switchable Berry curvature dipole in the monolayer topological insulator $\mathrm{WTe}_{2}$. Nat. Phys. 14, 900-906 (2018).

10. Sajadi, E. et al. Gate-induced superconductivity in a monolayer topological insulator. Science 362, 922-925 (2018).

11. Hou, W. et al. Strain-based room-temperature non-volatile $\mathrm{MoTe}_{2}$ ferroelectric phase change transistor. Nat. Nanotechnol. 14, 668-673 (2019).

12. $\mathrm{Yu}, \mathrm{W}$. et al. Chemically exfoliated $\mathrm{VSe}_{2}$ monolayers with room-temperature ferromagnetism. Adv. Mater. 31, 1903779 (2019).

13. Choi, Y.-B. et al. Evidence of higher-order topology in multilayer WTe $\mathrm{C}_{2}$ from Josephson coupling through anisotropic hinge states. Nat. Mater. 19, 974-979 (2020).

14. Wang, W. et al. Evidence for an edge supercurrent in the Weyl superconductor MoTe 2 . Science 368, 534-537 (2020).

15. Ali, M. N. et al. Large, non-saturating magnetoresistance in $\mathrm{WTe}_{2}$. Nature 514, 205-208 (2014).

16. Liu, W. et al. Enhanced superconductivity in the Se-substituted 1T-PdTe 2 . Phys. Rev. Mater. 5, 014802 (2021).

17. $\mathrm{Wu}, \mathrm{H}$. et al. Spacing dependent and doping independent superconductivity in intercalated 1T two dimensional $\mathrm{SnSe}_{2}$. 2D Mater. 6, 045048 (2019). 
18. Zhang, C. et al. Strain distributions and their influence on electronic structures of $\mathrm{WSe}_{2}-\mathrm{MoS}_{2}$ laterally strained heterojunctions. Nat. Nanotechnol. 13, 152-158 (2018).

19. Edelberg, D. et al. Approaching the intrinsic limit in transition metal diselenides via point defect control. Nano Lett. 19, 4371-4379 (2019).

20. $\mathrm{Yu}, \mathrm{Z}$. et al. Analyzing the carrier mobility in transition-metal dichalcogenide $\mathrm{MoS}_{2}$ field-effect transistors. Adv. Funct. Mater. 27, 1604093 (2017).

21. Lin, Z. et al. Defect engineering of two-dimensional transition metal dichalcogenides. 2D Mater. 3, 022002 (2016).

22. Wang, $X$. et al. Deriving $2 D M_{2} X_{3}(M=M o, W, X=S, S e)$ by periodic assembly of chalcogen vacancy lines in their MX 2 counterparts. Nanoscale 12, 8285-8293 (2020).

23. Liang, Q., Zhang, Q., Zhao, X., Liu, M. \& Wee, A. T. S. Defect engineering of twodimensional transition-metal dichalcogenides: applications, challenges, and opportunities. ACS Nano 15, 2165-2181 (2021).

24. Liu, E. et al. Giant anomalous Hall effect in a ferromagnetic kagome-lattice semimetal. Nat. Phys. 14, 1125-1131 (2018).

25. Little, A. et al. Three-state nematicity in the triangular lattice antiferromagnet $\mathrm{Fe}_{1}$, ${ }_{3} \mathrm{NbS}_{2}$. Nat. Mater. 19, 1062-1067 (2020).

26. $\mathrm{Zhu}, \mathrm{H}$. et al. New $\mathrm{Mo}_{6} \mathrm{Te}_{6}$ Sub-nanometer-diameter nanowire phase from $2 \mathrm{H}$ $\mathrm{MoTe}_{2}$. Adv. Mater. 29, 1606264 (2017).

27. Tiwari, R. K., Yang, J., Saeys, M. \& Joachim, C. Surface reconstruction of $\mathrm{MoS}_{2}$ to $\mathrm{Mo}_{2} \mathrm{~S}_{3}$. Surf. Sci. 602, 2628-2633 (2008).

28. Avsar, A. et al. Defect induced, layer-modulated magnetism in ultrathin metallic $\mathrm{PtSe}_{2}$. Nat. Nanotechnol. 14, 674-678 (2019).

29. Zhang, J. et al. Distorted monolayer $\mathrm{ReS}_{2}$ with low-magnetic-field controlled magnetoelectricity. ACS Nano 13, 2334-2340 (2019).

30. Jung, Y., Zhou, Y. \& Cha, J. J. Intercalation in two-dimensional transition metal chalcogenides. Inorg. Chem. Front. 3, 452-463 (2016).

31. Zhang, Q., Mei, L., Cao, X., Tang, Y. \& Zeng, Z. Intercalation and exfoliation chemistries of transition metal dichalcogenides. J. Mater. Chem. A 8, 15417-15444 (2020).

32. Wang, Z., Li, R., Su, C. \& Loh, K. P. Intercalated phases of transition metal dichalcogenides. SmartMat 1, e1013 (2020).

33. Novello, A. M. et al. Stripe and short-range order in the charge density wave of 1T- $\mathrm{Cu}_{\mathrm{x}} \mathrm{TiSe}_{2}$. Phys. Rev. Lett. 118, 017002 (2017).

34. Liao, J.-H., Zhao, Y.-C., Zhao, Y.-J., Yang, X.-B. \& Chen, Y. Doping induced charge density wave in monolayer $\mathrm{TiS}_{2}$ and phonon-mediated superconductivity. J. Appl. Phys. 127, 044301 (2020).

35. Bhoi, D. et al. Interplay of charge density wave and multiband superconductivity in $2 \mathrm{H}-\mathrm{Pd}_{\mathrm{x}} \mathrm{TaSe}_{2}$. Sci. Rep. 6, 24068 (2016).

36. Zhu, L. et al. Superconductivity in potassium-intercalated $\mathrm{T}_{\mathrm{d}}-\mathrm{WTe} \mathrm{e}_{2}$. Nano Lett. 18 6585-6590 (2018).

37. Nair, N. L. et al. Electrical switching in a magnetically intercalated transition metal dichalcogenide. Nat. Mater. 19, 153-157 (2020).

38. Sanikop, R., Budumuru, A. K., Gautam, S., Chae, K. H. \& Sudakar, C. Robust ferromagnetism in Li-intercalated and-deintercalated $\mathrm{MoS}_{2}$ nanosheets: implications for 2D spintronics. ACS Appl. Nano Mater. 3, 11825-11837 (2020).

39. Yim, C. et al. High-performance hybrid electronic devices from layered $\mathrm{PtSe}_{2}$ films grown at low temperature. ACS Nano 10, 9550-9558 (2016).

40. Chia, X. et al. Layered platinum dichalcogenides $\left(\mathrm{PtS}_{2}, \mathrm{PtSe}_{2}\right.$, and $\left.\mathrm{PtTe}_{2}\right)$ electrocatalysis: monotonic dependence on the chalcogen size. Adv. Funct. Mater. 26, 4306-4318 (2016).

41. Wang, Z., Li, Q., Besenbacher, F. \& Dong, M. Facile synthesis of single crystal PtSe nanosheets for nanoscale electronics. Adv. Mater. 28, 10224-10229 (2016).

42. Li, Y. et al. Topological origin of the type-II Dirac fermions in PtSe 2 . Phys. Rev. Mater. 1, 074202 (2017)

43. Zhang, K. et al. Experimental evidence for type-II Dirac semimetal in $\mathrm{PtSe}$. Phys. Rev. B 96, 125102 (2017).

44. Zhao, Y. et al. High-electron-mobility and air-stable 2D layered PtSe ${ }_{2}$ FETs. Adv. Mater. 29, 1604230 (2017).

45. Yao, W. et al. Direct observation of spin-layer locking by local Rashba effect in monolayer semiconducting $\mathrm{PtSe}_{2}$ film. Nat. Commun. 8, 14216 (2017).

46. Wang, Y. et al. Monolayer $\mathrm{PtSe}_{2}$, a new semiconducting transition-metal-dichalcogenide, epitaxially grown by direct selenization of Pt. Nano Lett. 15, 4013-4018 (2015).

47. Zheng, $H$. et al. Visualization of point defects in ultrathin layered $1 \mathrm{~T}-\mathrm{PtSe} \mathrm{e}_{2} .2 D$ Mater. 6, 041005 (2019)

48. Shawkat, M. S. et al. Thickness-independent semiconducting-to-metallic conversion in wafer-scale two-dimensional $\mathrm{PtSe}_{2}$ layers by plasma-driven chalcogen defect engineering. ACS Appl. Mater. Interfaces 12, 14341-14351 (2020).
49. Absor, M. A. U. et al. Defect-induced large spin-orbit splitting in monolayer $\mathrm{PtSe}_{2}$. Phys. Rev. B 96, 115128 (2017).

50. Sajjad, M., Singh, N. \& Schwingenschlögl, U. Strongly bound excitons in monolayer $\mathrm{PtS}_{2}$ and $\mathrm{PtSe}_{2}$. Appl. Phys. Lett. 112, 043101 (2018).

51. $\mathrm{Xu}, \mathrm{H}$. et al. Controlled doping of wafer-scale $\mathrm{PtSe}_{2}$ films for device application Adv. Funct. Mater. 29, 1805614 (2019).

52. Hikami, S., Larkin, A. I. \& Nagaoka, Y. Spin-orbit interaction and magnetoresistance in the two-dimensional random system. Prog. Theor. Phys. 63, 707-710 (1980)

53. Perdew, J. P., Burke, K. \& Ernzerhof, M. Generalized gradient approximation made simple. Phys. Rev. Lett. 77, 3865 (1996).

\section{ACKNOWLEDGEMENTS}

This work at the University of Texas at Dallas is supported by the US Air Force Office of Scientific Research grant no. FA9550-19-1-0037. This project is also partially funded by NSF- DMREF- 1921581, and the University of Texas at Dallas Office of Research through the Seed Program for Interdisciplinary Research (SPIRe) and Core Facility Voucher Program. J.Y.C. also acknowledges partial support from NSF-DMR-1700030. Work at Rice University was supported by the Gordon and Betty Moore Foundation's EPiQS Initiative through grant No. GBMF9470, the Robert A. Welch Foundation grant no. C-2024, the Alfred P. Sloan Foundation, as well as in part by NSF-DMREF-1921847. Work at UC Berkeley is funded by NSF-DMR-1921798. This research used resources of the Advanced Light Source, a U.S. DOE Office of Science User Facility under contract no. DE-AC02-05CH11231.

\section{AUTHOR CONTRIBUTIONS}

B.L. conceived and initiated this project, S.L., H.W., H.Z., W.L. and D.P.P. performed the sample growth and transport data measurements, Y.Z. and J.S.O. performed the ARPES experiments with the help of J.D. under the guidance of R.J.B. and M.Y., G.T.M. performed the single-crystal X-ray diffraction experiments, G.L. performed the theoretical calculations. S.L., M.Y. and B.L. wrote the paper with input from all authors.

\section{COMPETING INTERESTS}

The authors declare no competing interests.

\section{ADDITIONAL INFORMATION}

Supplementary information The online version contains supplementary material available at https://doi.org/10.1038/s41535-021-00382-x.

Correspondence and requests for materials should be addressed to Ming Yi or Bing Lv.

Reprints and permission information is available at http://www.nature.com/ reprints

Publisher's note Springer Nature remains neutral with regard to jurisdictional claims in published maps and institutional affiliations.

Open Access This article is licensed under a Creative Commons Attribution 4.0 International License, which permits use, sharing, adaptation, distribution and reproduction in any medium or format, as long as you give appropriate credit to the original author(s) and the source, provide a link to the Creative Commons license, and indicate if changes were made. The images or other third party material in this article are included in the article's Creative Commons license, unless indicated otherwise in a credit line to the material. If material is not included in the article's Creative Commons license and your intended use is not permitted by statutory regulation or exceeds the permitted use, you will need to obtain permission directly from the copyright holder. To view a copy of this license, visit http://creativecommons. org/licenses/by/4.0/.

(c) The Author(s) 2021 